\title{
Smart Cities no Brasil e em Portugal: o estado da arte
}

Smart Cities in Brazil and Portugal: the state of the art

Maria Abadia Alves[a], Ricardo Cunha Dias $[$ [b] (1), Paulo Castro Seixas $[b]$ (1)

[a] Ministério da Economia do Brasil, Secretaria de Coordenação e Governança do Patrimônio da União, Brasília, DF, Brasil

[b] Universidade de Lisboa (UL), Instituto Superior de Ciências Sociais e Políticas (ISCSP), Lisboa, Portugal

Como citar: Alves, M. A., Dias, R. C., \& Seixas, P. C. (2019). Smart Cities no Brasil e em Portugal: o estado da arte. urbe. Revista Brasileira de Gestão Urbana, 11, e20190061. https://doi.org/10.1590/2175-3369.011. e20190061

\section{Resumo}

Este texto analisa o estado da arte das Smart Cities no Brasil e em Portugal, procurando mapear e caracterizar os projetos existentes, identificar os serviços/setores objeto de inovação e os principais constrangimentos e desafios nos dois países. Recorrendo a uma revisão da literatura sobre o tema, os resultados mostram que, apesar de as Smart Cities configurarem já uma política de modelação urbana, evidenciada por índices e rankings, ambos os países parecem não ter ultrapassado uma primeira fase (Smart Cities 1.0), ou seja, de infraestruturação tecnológica. Os exemplos de tecnologia integrada como suporte de planejamento estratégico urbano (Smart Cities 2.0) é incipiente e a cocriação urbana pelos habitantes e city-users (Smart Cities 3.0) reduz-se a casos.

Palavras-chave: Smart Cities. Portugal. Brasil. Políticas de Cidade.

\section{Abstract}

This paper analyzes the state of the art of the Smart Cities in Brazil and Portugal, aimed at mapping and characterizing existing projects, identifying the services/sectors that are the object of innovation and the main constraints and challenges in each country. Based on a review of the literature on the subject, the main results showed that although the Smart Cities already have an urban modeling policy, evidenced by indexes and rankings, both countries did not seem to have passed a first phase (Smart Cities 1.0), or technological infrastructures. Examples of integrated technology as a support for urban strategic planning (Smart Cities 2.0) are incipient, and urban co-creation by the inhabitants and city-users (Smart Cities 3.0) is reduced to cases.

Keywords: Smart Cities. Portugal. Brazil. City Policies.

MAA é doutora em Economia Aplicada, e-mail: maria.abadia@planejamento.gov.br

RCD é mestre em Sociologia, e-mail: rdias@iscsp.ulisboa.pt

PCS é doutor em Antropologia, e-mail: pseixas@iscsp.ulisboa.pt 


\section{Introdução}

As Smart Cities (Cidades Inteligentes) configuram hoje uma importante política de modelação de desenvolvimento urbano sustentável (Seixas, 2019). 0 conceito associa as oportunidades da revolução digital, como a crescente difusão e capacidade de computação das novas tecnologias, à inovação social e à integração de mecanismos e ações de gestão e planejamento de espaços urbanos (Cunha et al., 2016). $\mathrm{Na}$ literatura, as classificações de smart cities existentes são geralmente apresentadas em diferentes estágios/gerações modelares entre um desenvolvimento urbano "technology-driven" e "citizen-co-creation" (Cohen, 2015), configurando polos em tensão (Selada, 2019) entre múltiplas fases intermédias de integração tecnológica ao nível da cidade (Leem \& Kim, 2013).

Na prática, as possibilidades/configurações da articulação entre TIC e gestão urbana expressam-se em uma grande diversidade de projetos difíceis de classificar, quer pelo nível/escala e o alcance/integração territorial das tecnologias adotadas, quer pelo grau/tipo de participação das comunidades locais. Para além disso, em alguns países esse movimento ainda não começou, e mesmo nos que já existem políticas de Smart Cities, a maioria encontra-se ainda em estágios embrionários marcados por projetos-piloto (Robinson, 2016).

No caso do Brasil e de Portugal, esses países parecem estar em um nível de potencial tecnológico similar para o desenvolvimento de Smart Cities. No entanto, a pouca literatura/evidência sobre as experiências já desenvolvidas nesses países configura uma lacuna para a qual este texto pretende contribuir. Fazendo uma revisão da literatura sobre o tema, a identificação de rankings e índices, bem como de projetos, o objetivo deste texto é justamente analisar o estado da arte das Smart Cities no Brasil e em Portugal. Para isso, colocaram-se as seguintes questões de partida:

1. Quais são as cidades "mais inteligentes" desses países?

2. Quais os serviços/setores objeto de inovação nessas cidades?

3. Quais os principais constrangimentos e desafios ao desenvolvimento de Smart Cities?

Numa primeira parte do artigo, analisa-se brevemente o conceito de 'smart cities', destacando algumas tendências de conceptualização. Numa segunda e última parte, procura-se, então, caracterizar a situação das Smart Cities no Brasil e em Portugal, identificando as "smartest cities", os serviços/setores objeto de inovação, bem como os constrangimentos e desafios em cada país.

\section{Referencial teórico}

As Smart Cities têm ocupado de forma crescente as discussões nos fóruns acadêmicos, mediáticos, empresariais e políticos (Mora et al., 2017). A este interesse, porém, não parece ter correspondido um maior esclarecimento sobre o termo. Com efeito, para além da multiplicidade de conceitos relacionados, tais como 'wired cities' (Dutton et al., 1987), 'cyber cities' (Graham \& Marvin, 1999), 'digital cities' (Ishida \& Isbister, 2000), 'intelligent cities' (Komninos, 2002), os quais parecem remeter para diferentes estágios tecnológicos (Leem \& Kim, 2013), são vários os trabalhos que têm procurado definir teoricamente 'smart cities' (Albino et al., 2015). Não se pretendendo fazer uma sumarização da extensa bibliografia sobre o assunto, parece, de qualquer forma, que o termo 'smart' está entre, por um lado, uma lógica de tecnopólis, pela utilização nas cidades de novas tecnologias (IoT ${ }^{1}$; big data ${ }^{2}$; governança algorítmica; etc.) e, por outro, de uma lógica de cidade inovadora, pela inclusão e participação cidadã na governança urbana.

Em um grande número de publicações, a integração de sistemas/serviços urbanos através das TIC é a característica definidora das Smart Cities como um modelo ideal. Na busca por alcançar tal modelo, o que se observa na prática é a priorização de setores urbanos e a eleição de focos de atenção

\footnotetext{
${ }^{1}$ Internet of Things (Internet das coisas): objetos/estruturas com capacidades infocomunicacionais avançadas.

${ }^{2}$ Macrodados ou grandes dados: grande conjunto/volume de dados gerados e armazenados pela utilização das TIC.
} 
(Cunha et al., 2016). Assim, em um dado momento, determinada cidade pode estar comprometida em melhorar uma das dimensões/setores da cidade, como, por exemplo, a área de mobilidade, introduzindo Sistemas e Serviços Inteligentes de Transporte, uma rede elétrica inteligente (smart grid) ou a implantação de sistemas de monitoramento urbano. É a partir desta priorização que se pode depois procurar sinergias entre os projetos existentes, para finalmente, em um estágio avançado, integrar tais iniciativas em um plano estratégico e de governança, com plataformas digitais abertas e informação ubíqua (INTELI, 2012; Leem \& Kim, 2013). As cidades, mediadas por 'tecnologias infiltradas', tornar-se-iam, assim, em um 'metaespaço ampliado e intensificado' pelas capacidades comunicativas e interativas entre pessoas e espaços (Duarte \& Firmino, 2011).

Tal mediação é importante desde logo porque a complexidade que caracteriza atualmente os

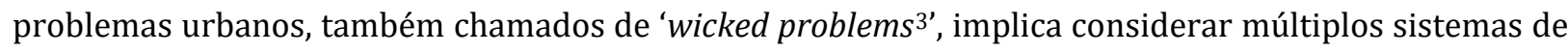
interação, um conhecimento imperfeito e incertezas sociais e institucionais (Goodspeed, 2015). Dado que as soluções tradicionais para esses problemas resultam, por norma, em novos problemas, isso representa um enorme desafio no quadro das atuais dinâmicas populacionais, econômicas e ambientais, e suas projeções. Como argumenta Goodspeed (2015), a visão dos problemas urbanos como 'wicked problems' requer monitorização de big data, a inovação local e a participação dos stakeholders (grupos de interesse). Desse modo, as 'smart cities' definem-se como cidades que criam as condições de governança, infraestruturas e tecnologia para produzir inovação social capaz de resolver problemas de crescimento, inclusão, sustentabilidade ambiental e qualidade de vida, através da escuta e do envolvimento dos vários atores locais: cidadãos, governos, universidades, empresas e associações.

Por essa razão, a chamada 'soft infrastructure' (governança em rede e fóruns de inovação) ganha importância para o desenvolvimento de indicadores e métodos de monitorização das dinâmicas urbanas, e as redes sociotécnicas, envolvendo os múltiplos atores da cidade na governança urbana, passam a configurar um determinante de sucesso das políticas públicas (Frey et al., 2007). Neste quadro, para além da infraestrutura tecnológica, algumas pesquisas têm-se centrado na importância da educação, capital humano e inovação social e colaborativa (Bencardino \& Greco, 2014), colocando as comunidades locais como indutores da transformação urbana pretendida (Seixas et al., 2017).

Assim, embora não exista uma definição inequívoca de 'smart city', identificam-se três abordagens (Bencardino \& Greco, 2014, p. 40): (1) 'tecnocentrada' (ênfase em hardware, uso e infraestrutura das TIC); (2) 'centrada nas pessoas' (ênfase na inovação social e capital humano); e (3) 'integrada' (integração dos dois pontos anteriores ao nível estratégico da cidade). No quadro desta última abordagem, as ideias de comunidades/cidades "inteligentes" e "sustentáveis" aparecem cada vez mais relacionadas. De fato, o conceito de 'smart cities' parece mesmo estar a ser substituído pelo de 'smart sustainable city' (cidade sustentável inteligente), definido pela International Telecommunication Union (ITU), a agência das Nações Unidas no campo das TIC, como

[...] uma cidade inovadora que utiliza as TIC e outros meios para melhorar a qualidade de vida, a eficiência da operação e serviços urbanos e a competitividade, garantindo as necessidades das gerações atuais e futuras em termos econômicos, sociais e ambientais (ITU, 2014, p. 13).

Esta evolução conceptual foi desenvolvida por Cohen (2015) através de três momentos/gerações de Smart Cities:

Smart Cities 1.0 - technology-driven (impulsionada pela tecnologia): os principais encorajadores dos projetos de smart cities são os provedores de serviços TIC, persuadindo as autoridades/gestores locais a adotarem as soluções por eles apresentadas.

\footnotetext{
${ }^{3}$ Wicked problems, termo traduzido como 'problemas complexos' ou 'problemas perversos'.
} 
Smart Cities 2.0 - technology-enabled, city-led (tecnologia habilitada, liderada pela cidade): os projetos são liderados pelas autoridades/gestores locais que passam a programar a implementação de tecnologias inteligentes e outras inovações em função de um futuro pretendido.

Smart Cities 3.0 - citizen co-creation (cocriação por cidadãos): os cidadãos assumem uma postura ativa e passam a ser os principais indutores e idealizadores dos projetos de inovação e de melhoria da qualidade de vida da cidade.

Apesar da integração idealizada, o relatório Black \& Veatch (2016) refere que a maioria dos projetos de smart cities são ainda essencialmente promovidos por governos locais, enquanto a integração de soluções inteligentes em outras escalas, como a metropolitana/regional, é menos comum. Robinson (2016) vai mais longe, dizendo que, passados vinte anos do surgimento do tema, a grande maioria das iniciativas continuam a ser projetos-piloto, com fontes de recursos geralmente limitadas a subvenções para I\&D, disponibilizadas por fundos internacionais ou recursos privados de empresas de tecnologia 4 Além disso, os investimentos em tecnologia inteligente visam principalmente usos comerciais, não sendo, portanto, suficientes para promover projetos na escala requerida para uma verdadeira transformação urbana e que, em muitos casos, os benefícios econômicos, sociais e ambientais são apenas uma colateralidade dos investimentos.

Assim, para Robinson (2016), medidas emanadas do setor público, como alterar as regras de licitações e contratos de forma a incluir critérios de cidades inteligentes, incluir nos contratos de infraestrutura incentivos ao desenvolvimento de tecnologia ou ainda condicionar o uso de fundos locais de investimento a empreendimentos relacionados às Smart Cities, poderiam ser bastante favoráveis. Estes são argumentos importantes para compreender a situação brasileira e portuguesa.

\section{As cidades mais inteligentes}

Brasil e Portugal são países lusófonos com realidades territoriais distintas, porém, ambos enfrentam desafios de urbanização semelhantes. Nos dois casos, a maioria dos habitantes conectada à Internet $\mathrm{e}$ familiarizada com o uso de TIC vive nas cidades, estando, assim, em estágios similares relativamente ao potencial de desenvolvimento de Smart Cities.

No Brasil, apesar da pouca bibliografia sobre o tema, alguns trabalhos têm procurado mapear as "smartest cities", apresentando, geralmente, metodologias variáveis eestabelecendo rankings e/ou índices.

Em 2011 e 2012, o Centro de Pesquisa e Desenvolvimento em Telecomunicações (CPqD) elaborou o "Índice Brasil de Cidades Digitais" a partir de um questionário sobre infraestrutura tecnológica (equipamentos, cobertura geográfica), disponibilidade de serviços digitais e recursos de acessibilidade para pessoas com deficiências físicas ou analfabetas. Em 2011, o questionário foi aplicado a 75 municípios, tendo sido alargado, em 2012, a 100 municípios (Quadro 1).

Mais recentemente, em 2016 e 2017, a empresa Urban Systems elaborou o ranking "Connected Smart Cities Brasil". Partindo de 3 dimensões (inteligência, conexão e sustentabilidade) e 11 indicadores (mobilidade, urbanismo, ambiente, energia, tecnologia e inovação, economia, educação, saúde, segurança, empreendedorismo e governança), o ranking classificou as três melhores cidades por porte/tamanho. São Paulo foi a primeira colocada entre as cidades com mais de 500 mil habitantes; Vitória ficou com a melhor posição entre 100 e 500 mil habitantes (5ำ lugar do ranking geral); e Cajamar, a melhor classificada até 100 mil habitantes (36olugar).

\footnotetext{
${ }^{4}$ No âmbito de fundos internacionais, destacam-se na União Europeia (EU) os fundos do Horizonte 2020 - Programa-Quadro Comunitário de Investigação \& Inovação, que em 2014 disponibilizou 92,32 milhões de euros para Smart Cities; e de fundos privados: a Cisco, empresa com representação no Brasil e em Portugal, que anunciou em 2019 um programa de US\$ 1 bilhão para o desenvolvimento de Smart Cities.
} 
Quadro 1 - Resultados Índice CPqD e Ranking Connected Smart Cities

\begin{tabular}{|c|c|c|c|c|}
\hline \multirow{2}{*}{ Posição } & \multicolumn{2}{|c|}{ Índice CPqD } & \multicolumn{2}{|c|}{ Ranking Connected Smart Cities } \\
\hline & 2011 & 2012 & 2016 & 2017 \\
\hline 1 & Belo Horizonte (MG) & Curitiba (PR) & São Paulo (SP) & São Paulo (SP) \\
\hline 2 & Curitiba (PR) & Rio de Janeiro (RJ) & Rio de Janeiro (RJ) & Curitiba (PR) \\
\hline 3 & Porto Alegre (RS) & Belo Horizonte (MG) & Curitiba (PR) & Rio de Janeiro (RJ) \\
\hline 4 & Vitória (ES) & Vitória (ES) & Brasilia (DF) & Belo Horizonte (MG) \\
\hline 5 & $\begin{array}{l}\text { Ibirapuitã (RS) e Jundiaí } \\
\text { (SP) }\end{array}$ & Campinas (SP) & Belo Horizonte (MG) & Vitória (ES) \\
\hline 6 & Campinas (SP) & $\begin{array}{c}\text { Sorocaba (SP) e } \\
\text { Anápólis (GO) }\end{array}$ & Vitória (ES) & Florianópolis (SC) \\
\hline 7 & $\begin{array}{c}\text { Santos (SP) e São Carlos } \\
\text { (SP) }\end{array}$ & Porto Alegre (RS) & Florianópolis (SC) & Brasilia (DF) \\
\hline 8 & Tarumã (SP) & Jundiaí (SP) & Barveri & Campinas (SP) \\
\hline 9 & São Paulo (SP) & Guarulhos (SP) & Recife (PE) & $\begin{array}{c}\text { São Caetano do Sul } \\
\text { (SP) }\end{array}$ \\
\hline 10 & Tauá (CE) & Santos (SP) & Campinas (SP) & Recife (PE) \\
\hline
\end{tabular}

Fonte: Adaptado de Telesíntese $(2011$, 2012) e Connected Smart Cities $(2015,2016)$.

Przeybilovicz et al. (2014) realizaram outro estudo sobre o perfil das cidades brasileiras no uso e infraestrutura de TIC. A pesquisa envolveu 5.565 municípios e informações disponíveis pela Pesquisa de Informações Básicas Municipais (Munic) realizada pelo Instituto Brasileiro de Geografia e Estatística (IBGE). Os elementos analisados foram: infraestrutura de TIC da prefeitura, infraestrutura municipal de acesso à Internet, e-Administração, e-Serviços, Comunicação com o público e inclusão digital, IDH-M, renda per capita e população. Da análise de clusters, surgiram os seguintes grupos:

Cluster 1 - Ricos e grandes: possuem boa infraestrutura de acesso à Internet, oferecem e-serviços aos cidadãos, e a prefeitura possui boa infraestrutura de TIC. Neste cluster estão 12 capitais estaduais (São Paulo, Rio de Janeiro, Manaus, Cuiabá, Natal e outras). A maioria das cidades deste cluster é da região Sudeste e Sul. Apesar de possuir bons recursos de infraestrutura e uso de TIC, apresentaram maus resultados na inclusão digital.

Cluster 2 - Pobres e sem tecnologia: corresponde ao grupo de municípios com piores indicadores de uso e infraestrutura de TIC. Não possuem infraestrutura de TIC nem de acesso à Internet, os serviços eletrônicos são maus, não promovem a inclusão digital e praticamente não utilizam as TIC na administração pública. 0 maior de número de cidades deste grupo está na região Nordeste, mas cidades pobres e sem tecnologia estão presentes em todas as regiões brasileiras.

Cluster 3 - Tecnológicos: são os municípios mais avançados no uso e infraestrutura de TIC. Todos os indicadores de TIC são os melhores, da infraestrutura ao uso para o cidadão. Neste cluster está o segundo grande grupo de capitais (12), entre elas Curitiba, Porto Alegre, Florianópolis, Belo Horizonte, Salvador, Fortaleza, Teresina. É um grupo maioritariamente formado por cidades do Sul e do Sudeste.

Cluster 4 - Medianos: são os municípios que possuem infraestrutura de acesso à Internet e de TIC na prefeitura e praticam a inclusão digital, mas que ainda precisam melhorar a oferta de e-serviços, a comunicação e a gestão e uso das TIC na administração pública. É o segundo cluster com mais municípios (810). As capitais Macapá e Maceió estão neste grupo.

Cluster 5 - Pequenos interessados: sendo pequenos, fazem uso da TIC na administração pública e possuem boa comunicação com o cidadão. Embora precisem melhorar, já oferecem e-serviços e mostramse preocupados com ações de inclusão digital. Este é o cluster com mais municípios (866). Grande parte deles é do Sudeste e Sul.

Cluster 6 - Medianos sem tecnologia: apresentam a pior comunicação com o cidadão, são maus com na inclusão digital e no acesso à Internet. Também precisam melhorar a oferta de e-serviços e infraestrutura de TIC na prefeitura. É grandemente formado por cidades da região Nordeste. 
Sem cluster - formado por 2.438 cidades (44\% do total de municípios brasileiros) foram excluídos da amostra por não cumprirem critérios de resposta. Desconhece-se a sua situação em relação ao uso e à infraestrutura de TIC.

Embora os trabalhos sintetizados apresentem metodologias diferentes, os resultados permitem inferir: a grande concentração espacial dos municípios; e uma predominância das capitais estaduais das regiões Sul e Sudeste na implementação de projetos de smart cities (o cluster 3 com os municípios mais bem colocados). Dos dois rankings, observa-se, ainda, uma predominância de capitais de estado nos primeiros cinco lugares (as exceções são Ibirapuitã, Jundiaí e Campinas nos 5o lugares em 2011 e 2012). Entre as não capitais, há uma predominância de cidades do interior do estado de São Paulo. Observa-se também que não há cidades da região Norte do país.

Em Portugal, por seu turno, o "Índice de Cidades Digitais", desenvolvido pela INTELI em duas edições (2012 e 2016), é o principal índice de cidades inteligentes. Em ambas foi utilizada uma amostra de municípios integrantes da Living Lab RENER - Rede para Inovação Urbana. A RENER (Rede Portuguesa de Cidades Inteligentes) foi criada em 2013 no âmbito do Programa Nacional de Mobilidade Elétrica (Mobi-E), tendo as cidades envolvidas funcionado como test-bed (banco de ensaio) para a introdução do veículo elétrico em Portugal (INTELI, 2019a). No primeiro momento, a RENER contava com a participação de 43 municípios. A partir de 2016, a rede passou a integrar a Associação Nacional de Municípios Portugueses (ANMP), com a criação da Seção de Cidades Inteligentes, integrando124 municípios (INTELI, 2016). 0 índice, nas suas duas versões, foi composto por cinco dimensões: governança, inovação, sustentabilidade, qualidade de vida e conectividade. A primeira edição configurou uma fase-piloto com 20 cidades da rede. Em 2016 o número de municípios foi ampliado para 36 (INTELI, 2012, 2016). No Quadro 2 apresentam-se os primeiros cinco das duas edições.

Quadro 2 - Resultado Global Índice de Cidades Digitais 2012-2016

\begin{tabular}{|c|c|c|}
\hline Posição & $\mathbf{2 0 1 2}$ & $\mathbf{2 0 1 6}$ \\
\hline 1 & Lisboa & Porto \\
\hline 2 & Almada & Águeda \\
\hline 3 & Cascais & Cascais \\
\hline 4 & Aveiro & Bragança \\
\hline 5 & Vila Nova de Gaia & Guimarães \\
\hline
\end{tabular}

Fonte: INTELI $(2012,2016)$.

Em 2012, para além da capital, os primeiros lugares foram ocupados por municípios das áreas metropolitanas de Lisboa e Porto e em cidades com forte presença universitária (Selada \& Silva, 2013). Em 2016, Lisboa sai dos primeiros 5 lugares e o Porto, sede da segunda maior área metropolitana do país, passa para a primeira posição. As demais cidades não constavam em 2012, à exceção de Cascais, e também com essa exceção são todas do norte de Portugal. Águeda, Bragança e Guimarães são cidades médias.

Para além deste índice, a empresa IDC Portugal, em colaboração com a NOVA IMS, publicou, em 2015, o estudo "Portuguese Smart Cities Index 2015", classificando 50 municípios em função do nível de desenvolvimento de infraestruturas e processos necessários para as Smart Cities do futuro. A metodologia sustentou-se na ponderação entre duas categorias: 1) "Forças Motrizes" (com as dimensões: Pessoas, Economia e TIC); e 2) "Vetores de Inteligência" (com as dimensões Smart Government, Smart Buildings, Smart Mobility, Smart Services \& Living, e Smart Energy \& Environment). 0 estudo identificou três clusters de municípios "inteligentes", classificados como (1) Top; (2) Competidores; e (3) Seguidores, sendo o primeiro grupo composto por Lisboa, Oeiras, Porto e Bragança. 


\section{Setores e serviços priorizados}

Relativamente ao Brasil, Cunha et al. (2016) realizaram, em 2015, uma pesquisa com recurso a uma amostra não representativa, com 1.664 respondentes de 14 municípios (29\%, habitantes de cidades médias, 33\% de grandes e 38\% de megalópoles). Os resultados aferiram 'Segurança', 'Saúde' e 'Educação' como os aspetos mais importantes de uma cidade inteligente. Tal deve ser tido em conta no futuro, dado que nenhum desses setores é de responsabilidade estritamente local. Assim, projetos que visem desenvolver ações nesses setores terão invariavelmente de contar com a colaboração de entidades supralocais, sejam os estados e/ou a União, implicando uma governança multinível.

Embora existam já iniciativas nesses três setores, a predominância de projetos observa-se no setor da energia. Isso pode ser explicado pela alteração introduzida com as Resoluções no 414/2010 (ANEEL, 2010) e 479/2012 (ANEEL, 2012) da Agência Nacional de Energia Elétrica (ANEEL), que transferiu das distribuidoras de energia para os municípios os ativos de iluminação pública. Ainda que a gestão do serviço de iluminação pública seja, constitucionalmente, uma competência municipal, a resolução obriga os municípios a receber esses ativos e a responsabilizar-se por projetos de ampliação, manutenção e modernização da iluminação pública de suas dependências (BNDES, 2018). Dadas as novas atribuições e o cenário de restrição fiscal vivenciado pelos municípios brasileiros, o uso de Parcerias Público-Privadas (PPP) tornou-se a principal opção no país para a viabilização de projetos de modernização da iluminação pública.

Até 2017 existiam já mais de 100 projetos de PPP iniciados pelas municipalidades para a implantação de sistemas de iluminação pública inteligente. Modelados a partir de concessões administrativas, o investimento médio de um contrato de PPP foi aproximadamente de 273 milhões de reais (Antunes, 2017 apud BNDES, 2018). É certo que a modernização da iluminação pode viabilizar outros serviços, pois a substituição de lâmpadas pela tecnologia LED (Light Emitting Diode) possibilita envolver inteligência na rede e tecnologias de sensoriamento no poste, dimerização e acionamento por movimentação e presença, alargando o escopo desses projetos. No entanto, trata-se de projetos contingentes dada esta nova realidade institucional que não são priorizados pela população, pois a modernização da rede pública não é diretamente central para os cidadãos (Cunha et al., 2016).

Ainda em relação a este setor, é de destacar o Sistema de Compensação de Energia Elétrica, relativo à micro e minigeração distribuída, que permite qualquer consumidor ser um produtor de energia, injetando o excedente na rede da distribuidora (Resolução Normativa ANEEL no. 482/2012). Quando este excedente é maior que a energia consumida, o consumidor-gerador recebe um crédito a ser utilizado para abater no consumo ou na fatura mensal (ANEEL, 2018). Esse sistema de incentivo ao envolvimento do cidadão pode colocar as Smart Cities em outro patamar de desenvolvimento (3.0) com ganhos econômicos e ambientais. Em 2017, o número de conexões de micro e minigeração de energia no Brasil ultrapassava as $10 \mathrm{mil}$, destacando-se fortemente a fonte solar. Os estados com mais micro e minigeradores eram o de Minas Gerais (2.225 conexões), São Paulo (2.094) e Rio Grande do Sul (1.096) (ANEEL, 2017).

Os municípios brasileiros têm também feito investimentos em outros projetos que podem tornar as cidades "mais inteligentes" (Quadro 3). Muitos desses projetos-"piloto" são financiados por empresas do setor das telecomunicações. 0 caso mais conhecido talvez seja o da cidade de Águas de São Pedro, município turístico do interior de São Paulo, que recebeu, a partir de 2014, um projetopiloto financiado pela Telefônica/Vivo, a maior provedora de serviços de telecomunicações do Brasil (Telefônica, 2015). 
Quadro 3 - Iniciativas de Smart Cities no Brasil

\begin{tabular}{|c|c|}
\hline Cidades & Iniciativas \\
\hline Fortaleza (CE) & $\begin{array}{l}\text { - Implementação de GPS em ônibus para maior previsibilidade nos itinerários. } \\
\text { - Projeto-piloto de partilha de carros elétricos através de loT. }\end{array}$ \\
\hline Águas de São Pedro (SP) & $\begin{array}{l}\text { - } 500 \text { sensores de estacionamento para informar, por meio de aplicativo, a disponibilidade de } \\
\text { vagas nas principais vias do município. } \\
\text { - Implantação de iluminação pública inteligente em um dos principais parques da cidade } \\
\text { para controlar o estado das lâmpadas e sua operação. } \\
\text { - Câmeras inteligentes de monitoramento das vias públicas, com geração de alarmes. } \\
\text { - Solicitação online de consultas médicas. } \\
\text { - Acesso biométrico dos usuários aos registros médicos e consultas, garantindo uma } \\
\text { comunicação confidencial. } \\
\text { - Dispositivos móveis nas escolas para alunos e professores. }\end{array}$ \\
\hline Rio de Janeiro (RJ) & $\begin{array}{l}\text { - Centro de Operações Rio (COR), fundado em 2010, integrando cerca de } 30 \text { agências da } \\
\text { cidade que acessam dados de câmeras e sensores, visando melhorar o trânsito e a gestão } \\
\text { de emergências na cidade. } \\
\text { - A região da Praça Mauá foi escolhida para as transformações do Programa de Inovação } \\
\text { Social e Urbana de empresa multinacional de conectividade. Ele apresenta } 15 \text { soluções } \\
\text { inteligentes desenvolvidas pela empresa e startups de tecnologia, e suas principais soluções } \\
\text { em loT incluem monitoramento da qualidade do ar, monitoramento e gerenciamento de } \\
\text { bueiros e sensores de ruído. }\end{array}$ \\
\hline Canoas (RS) & $\begin{array}{l}\text { - Implantação de mais de } 30 \text { sensores para detectar ruídos de alerta, como disparos de } \\
\text { armas de fogo, que avisam automaticamente a Central Integrada de Monitoramento do } \\
\text { Gabinete de Gestão Integrada Municipal. }\end{array}$ \\
\hline Paulínia (SP) & $\begin{array}{l}\text { - Instalação de } 25 \text { estações de coleta de lixo na cidade, reduzindo até } 30 \% \text { os custos desse } \\
\text { serviço. }\end{array}$ \\
\hline São José dos Campos (SP) & $\begin{array}{l}\text { - Implantação de sensores climáticos e de detecção de disparos e de ruídos, por exemplo, } \\
\text { para medir temperatura, umidade e níveis de CO2. } \\
\text { A cidade também se beneficiará: } \\
\text { - de uma rede de Wi-Fi pública; } \\
\text { - de um sistema de iluminação pública inteligente; } \\
\text { - de um sistema de resposta de emergência, composto por } 500 \text { câmeras conectadas; } \\
\text { - de sistemas de software e de } 205 \text { km de cabos de fibra ótica. }\end{array}$ \\
\hline Itu (SP) & $\begin{array}{l}\text { - Implantação de um sistema inteligente de coleta de resíduos, com } 3.300 \text { contêineres } \\
\text { distribuídos pela cidade. }\end{array}$ \\
\hline Vitória (ES) & $\begin{array}{l}\text { - Implantação da Rede Bem-Estar, que interliga os equipamentos de saúde (unidades de } \\
\text { saúde, pronto-atendimentos, farmácias, laboratórios, consultórios odontológicos, centros de } \\
\text { referência e especialidades) em um único sistema. } \\
\text { - Implantação do "Prontuário Eletrônico", software que oferece senviços como o de gestão } \\
\text { de agendamento de retorno de consultas e a avaliação de atendimento via SMS. }\end{array}$ \\
\hline
\end{tabular}

Fonte: Adaptado de Cunha et al. (2016) e Telefônica (2015).

A Algar Telecom, uma empresa local de telecomunicações, tem também um projeto de implantação de um bairro inteligente (bairro da Granja Marileusa) na cidade de Uberlândia (MG), criado para receber aplicações de IoT, com infraestrutura de rede de energia e dados, oito dutos de telefonia e redundância, e tem mais de 95 casas com monitoramento por vídeo e fibra ótica instalada. Equipado de lixeiras com sensores de volume, o bairro originou ainda um micropolo tecnológico e possui espaço de coworking para atrair empresas inovadoras (ITF365, 2017).

Já o projeto Smart City Laguna, criado em Croatá (CE) em 2011, é também de iniciativa privada e pretende ser a primeira 'cidade inteligente social' do mundo, baseando-se nos pilares de inclusão social, planejamento urbano, meio ambiente e tecnologia. Com a construção de casas apoiadas pelo programa Minha Casa, Minha Vida ${ }^{5}$ o bairro prevê o uso gratuito de tecnologias que obtêm informações de base local e o monitoramento de recursos como água e energia. 0 projeto possui já parceiros privados para o fornecimento de medidores inteligentes, postes inteligentes, sinal gratuito de Wi-Fi e sistemas de segurança (Smart City Laguna, 2019).

\footnotetext{
${ }^{5}$ Iniciativa do Governo Federal do Brasil lançada em 2009 que visa subsidiar/auxiliar a aquisição de casa, levando em conta a renda das famílias.
} 
Por fim, o Sebrae, uma instituição privada sem fins lucrativos que tem por missão auxiliar a competitividade e sustentabilidade de pequenos empreendimentos, lançou, em 2016, um projeto-piloto cujo objetivo é estimular a interligação de dados, acessibilidade, mobilidade urbana, segurança e sustentabilidade nas cidades. Atualmente, são apoiados projetos em sete cidades: Campina Grande, Campinas, Curitiba, Florianópolis, Maceió, Maringá e Vitória. Até 2019, o Sebrae investirá R\$ 10 milhões nesses projetos (Rede Brasileira de Cidades Inteligentes e Humanas, 2019).

Quanto a Portugal, a principal inciativa com maior participação municipal está relacionada com a mobilidade, através da implantação do projeto Mobi-E. Criado em 2009, o projeto visa promover a mobilidade pessoal elétrica por meio de ações que incentivem a adoção de veículos elétricos e a construção da infraestrutura necessária para recarregá-los. Nesse momento, o projeto abrange mais de 50 municípios, ultrapassando os 1.250 pontos de carregamento (Mobi.E, 2019).

Cascais e Porto apresentaram, em 2016, o maior número de utilizadores de carros elétricos inseridos na rede Mobi-E. Em relação à percentagem de novos automóveis elétricos comercializados sobre o total de automóveis adquiridos em 2015, destacam-se os municípios de Esposende, Santarém, Leiria, Aveiro, Braga e Porto (INTELI, 2016). Vila Nova de Gaia, Cascais e Sintra são os municípios com maior número de pontos de carregamento. Castelo Branco, Portalegre e Beja apresentavam a maior proporção de pontos de carregamento, em face ao total de veículos elétricos registrados no município em 2016 (INTELI, 2016).

Tal como no Brasil, os projetos de iluminação pública em Portugal têm também ganhado destaque em vários municípios. Porém, diferentemente do caso brasileiro em que o principal motivador é o imperativo da lei, no caso português, o principal indutor é a necessidade de diminuir os custos com o serviço. Aqui, devem ser tidos em conta os requisitos regulamentares para o acesso aos fundos da Política de Coesão da União, Europeia (UE), de que Portugal é Estado-membro. Sendo o Crescimento Inteligente um dos eixos da Estratégia Europa 2020 e a sustentabilidade e eficiência no uso de recursos e a modernização da administração pública dois dos domínios temáticos do atual Quadro Comunitário de Apoio (2014-2020), as iniciativas de modernização ecológica são uma das formas de os municípios mais facilmente acederem a fundos comunitários (Dias \& Seixas, 2018).

Segundo os resultados do "Índice de Cidades Digitais" (INTELI, 2016), 44\% dos municípios possuem também regulação de fluxo luminoso, e 39\% detêm sistemas de gestão da iluminação pública. Assim, embora muitas das iniciativas de iluminação pública se constituam como projetos-piloto financiados por fundos europeus e empresas privadas, há algumas que ultrapassaram já essa fase ou que tiveram desde o início o objetivo de fazer uma substituição completa do serviço tradicional por um mais econômico através da tecnologia LED, utilizando recursos próprios. Apesar de envolverem um investimento inicial significativo, os resultados com a queda dos gastos são relativamente rápidos. Isso, combinado com a estabilização da tecnologia LED, tende a incentivar os investimentos também com recursos orçamentários próprios (Público, 2016).

Quadro 4 - Projetos premiados com o selo "A Smart Project for Smart Cities" 2015

\begin{tabular}{|c|c|c|}
\hline Cidades & $\begin{array}{c}\text { Iniciativas } \\
\text { Gaila Nova de } \\
\text { Gaia }\end{array}$ & $\begin{array}{c}\text { Projeto NoPaper: visa à desmaterialização do processo de licenciamento urbanístico através } \\
\text { da disponibilização virtual de informação e ferramentas para a instrução em formato digital } \\
\text { de processos correspondentes a operações urbanísticas de urbanização, edificação ou outros } \\
\text { procedimentos conexos. }\end{array}$ \\
\hline Torres Vedras & $\begin{array}{c}\text { Projeto bike sharing: projeto de mobilidade urbana que disponibiliza bicicletas aos usuários a } \\
\text { partir de } 11 \text { bikestations situadas em vários locais da cidade, } 260 \text { bicicletas convencionais, } 30 \\
\text { bicicletas elétricas e software de gestão. }\end{array}$ \\
\hline $\begin{array}{c}\text { Sistema de gestão e informação ambiental: visa promover a conservação e valorização } \\
\text { ambiental dos espaços naturais classificados e incrementar a sua biodiversidade, através de } \\
\text { um sistema de informação que opera em uma componente infraestrutural, que envolve a } \\
\text { intervenção para a conservação de espaços naturais através da instalação de um sistema de } \\
\text { gestão e informação ambiental, na base tecnológica do Sistema de lnformação Geográfica } \\
\text { Municipal. }\end{array}$ \\
\hline Cascais & $\begin{array}{c}\text { Gestão inteligente de resíduos: integra duas tecnologias inovadoras para a gestão de resíduos } \\
\text { produzidos no município, uma de gestão de resíduos e outra que utiliza sondas de leitura do } \\
\text { nível de enchimento e comunicação à distância. A combinação dessas duas tecnologias } \\
\text { permite: planejamento e otimização dos circuitos de recolha; visualização dos circuitos nas } \\
\text { viaturas, através de computadores de bordo; registro automático das recolhas dos } \\
\text { contentores; registro de anomalias quando da recolha de resíduos indiferenciado e seletivo. }\end{array}$ \\
\hline
\end{tabular}

Fonte: Adaptado de INTELI (2016) e Câmara Municipal de Torres Vedras (2013). 
Portugal, como no Brasil, conta ainda com outras experiências de projetos de smart cities. 0 "Índice de Cidades Digitais" (INTELI, 2012, 2016) apresenta uma extensa lista de iniciativas em curso nas áreas da governança, inovação, sustentabilidade, qualidade de vida e conectividade. A INTELI (2019b) promove também o selo "A Smart Project for Smart Cities", atribuído a projetos de desenvolvimento urbano com soluções inovadoras e inteligentes (Quadro 4).

Por fim, em Portugal, tal como no Brasil, há um projeto de implantação de uma Smart City (PlanIT Valley) próxima ao Porto, idealizado de raiz, mas sem ter ainda saído do estádio de concepção (Smart City Hub, 2018).

\section{Principais constrangimentos e desafios}

No Brasil, dada a crise fiscal do setor público e a escassez de recursos orçamentários, a falta de financiamento é um dos principais constrangimentos a uma política de smart cities. Embora as PPP tenham aumentado significativamente com a necessidade de se modernizar a iluminação pública municipal, há ainda bastantes reticências em relação a este modelo. Como os contratos envolvem valores altos e prazos longos, estes precisam ser bem elaborados, e a maioria dos municípios não tem essa capacidade técnica, o que pode deixar a administração pública refém por décadas (Cunha et al., 2016).

Assim, a baixa capacidade técnica dos municípios dificulta a constituição de PPP, sendo também um entrave aos projetos de smart cities, pois toda contratação pública implica um documento técnico delimitando a pretensão pública (BNDES, 2018). Geralmente denominados Termos de Referência, estes documentos indicam as características relevantes a determinada TIC (em requisitos como interoperabilidade, cibersegurança, etc.) e promovem a competitividade. A ausência de conhecimento por parte do gestor público municipal é, portanto, um obstáculo para uma contratação eficiente (BNDES, 2018).

Outro aspeto relevante é a baixa capacidade de planejamento de longo prazo do setor público brasileiro. Como a implantação de projetos de smart cities requer uma visão de futuro, isso choca com a pouca tradição em planejamento de longo prazo no país, sendo que as políticas públicas são grandemente influenciadas pelos ciclos eleitorais. Ou seja, é comum acontecer, em qualquer dos níveis de governo (Federal, Estadual ou Municipal), que projetos de êxito sejam abandonados pelo governante eleito em pleito seguinte apenas porque foi elaborado na gestão de um governo opositor.

Finalmente, deve-se ressaltar o baixo envolvimento do Governo Federal nos projetos de smart city. Devido à peculiar organização federativa brasileira e à complexidade dos problemas urbanos atuais, o desenvolvimento desses projetos requer uma colaboração estreita dos três níveis governamentais. Em relação ao fomento das sinergias que ocorrem em áreas de predomínio Federal ou Estadual nos Municípios (por exemplo, entre mobilidade e saúde), seria importante desenvolver a ferramenta da Avaliação de Impacto Regulatório (AIR): processo que procura, a partir da definição de um problema regulatório, avaliar os possíveis impactos das opções regulatórias disponíveis para o alcance dos objetivos pretendidos (ANVISA, 2019). Aqui, o reforço a que se tem assistido para a eliminação da carga regulatória nos projetos de smart cities é relevante, mas não suficiente (ANATEL, 2018). É necessário que a AIR se constitua como um instrumento de verdadeira reflexão sobre a atuação e investigação necessárias para potenciar a transversalidade/integração de projetos de smart cities, e não apenas como uma ferramenta comparativa de opções regulatórias. Avaliar os impactos de políticas de smart cities de mobilidade urbana no sistema de saúde é um exemplo de uma 'abordagem para a transversalidade' (Smart Cities 2.0) que a AIR pode promover.

No caso português, o financiamento dos projetos é também uma questão central. Como observa Catarina Selada: “[...] não há nos orçamentos municipais grande margem de manobra e, como as soluções são pouco maduras, também o investimento privado é ainda cauteloso" (Público, 2016). No entanto, diferentemente do Brasil, os municípios portugueses têm a possibilidade de acessar fundos europeus. Selada \& Silva (2013) sumarizam para o período 2014-2020 os fundos disponíveis para projetos de cidades inteligentes, totalizando 418.249 M€. Apesar de existentes, esses recursos 
não são diretamente relacionados aos projetos de smart cities (Quadro 5). Para Antônio Almeida Henriques, vice-presidente da ANMP, na preparação do Portugal 2030 é preciso que o tema ganhe centralidade e passe a incluir os meios necessários para o desenvolvimento das smart cities de forma integrada e explícita (SapoTek, 2018).

Além disso, o acesso aos fundos requer uma capacidade técnica que pode não existir nos municípios. Essa ausência pode, como no Brasil, dificultar a contratação de projetos de cidades inteligentes mesmo quando estes são desenvolvidos às expensas do orçamento do próprio município. Essa fragilidade pode ser ultrapassada pela formação de consórcios intermunicipais e a ampliação das redes colaborativas já existentes.

Por fim, a territorialização do financiamento comunitário em Portugal, segundo a atual lógica europeia, tem evidenciado tensões de alinhamento entre agendas de desenvolvimento entre o nível europeu, nacional, regional e local (Ferreira \& Seixas, 2017). Em resultado do alto nível de centralização, setorialização e polarização da administração pública portuguesa e da inexistência de um verdadeiro nível regional de administração/planejamento, é possível que projetos como os de iluminação inteligente sejam apenas uma forma de os municípios maximizarem o acesso a fundos comunitário (Dias \& Seixas, 2018). Ou seja, sem um racional estratégico regional, essas iniciativas dificilmente poderão, no futuro, ampliar-se para um nível supralocal, configurando um desafio à passagem destes projetos para Smart Cities 2.0 e 3.0.

Quadro 5 - Programas com relevo para smart cities em 2014-2020

\begin{tabular}{|c|c|c|c|}
\hline Programas & Orçamento & "Climate earmarking" & Descrição \\
\hline Horizonte 2020 & $87.000 \mathrm{M} €$ & $35 \%$ & $\begin{array}{l}\text { Apoio à atividade de investigação e inovação, } \\
\text { com foco em áreas como: energia eficiente, } \\
\text { segura e limpa; transportes inteligentes, verdes e } \\
\text { integrados; ação climática e eficiência de } \\
\text { recursos. Irão ser abertos apelos específicos de } \\
\text { propostas para smart cities. }\end{array}$ \\
\hline COSME & $2.500 M €$ & n.a. & $\begin{array}{l}\text { Apoio à competitividade e sustentabilidade das } \\
\text { empresas europeias, com foco nas PMEs. Pode ser } \\
\text { utilizado para suportar o desenvolvimento de } \\
\text { competências e a emergência e expansão de } \\
\text { empresas fornecedoras de soluções para smart cities. }\end{array}$ \\
\hline $\begin{array}{l}\text { Política de } \\
\text { Coesão } \\
\text { (FEDER, FSE) }\end{array}$ & $325.149 M €$ & $\begin{array}{l}\text { 20\% do FEDER em cada } \\
\text { EM em regiões } \\
\text { desenvolvidas/em } \\
\text { transição e } 6 \% \text { em } \\
\text { regiões menos } \\
\text { desenvolvidas (apenas } \\
\text { eficiência } \\
\text { energética/energias } \\
\text { renováveis) }\end{array}$ & $\begin{array}{l}\text { Apoio a projetos de } 11 \text { objetivos temáticos } \\
\text { predefinidos, onde se integram: transição para } \\
\text { uma economia hipocarbônica; adaptação às } \\
\text { alterações climáticas, e prevenção de riscos, } \\
\text { transportes sustentáveis, ecoinovação em PMEs. }\end{array}$ \\
\hline LIFE+ & $3.600 M €$ & $902 M €$ & $\begin{array}{l}\text { A componente das alterações climáticas do } \\
\text { programa pode ser utilizada para promover } \\
\text { atividades de adaptação e mitigação, incluindo nas } \\
\text { áreas urbanas. Foca-se em projetos-piloto, boas } \\
\text { práticas, ações de disseminação e informação, etc. }\end{array}$ \\
\hline
\end{tabular}

Fonte: Selada \& Silva (2013).

\section{Conclusões}

Brasil e Portugal parecem estar em estágios similares no que diz respeito a uma política de smart cities. Em ambos os países, projetos-piloto pouco replicáveis e integrados em plataformas únicas de gestão são a principal característica. Uma exceção no caso português é a área da mobilidade elétrica (projeto Mobi-E), atualmente difundida em todo o país.

Assim, se adotarmos as três gerações de uma política de smart cities, podemos dizer que ambos os países estão ainda numa primeira geração (Smart Cities 1.0), ou seja, as Smart Cities, enquanto políticas 
de modelação urbana, estão ainda numa fase de pré-ação, configurando projetos mais ou menos isolados e muito associada a processos tecnológicos. A agregação de tais projetos numa perspectiva unificada de gestão, possibilitando uma verdadeira política de modelação urbana que evidencie uma visão estratégica de cidade (Smart Cities 2.0), a existir, encontra-se ainda num estágio de agendamento, configurando casos de estudo a ter em conta em investigações em curso.

Uma lógica de cocriação urbana de base cidadã (Smart Cities 3.0) sustentada por processos tecnológicos de acesso cidadão pleno, e cuja informação ("big" and "small" data) por estes inserida serve ao contínuo fazer cidade numa visão de planejamento estratégico partilhada em tempo real, é ainda apenas uma idealização. Em Portugal, está prevista em 2019 a criação de uma Plataforma de Gestão Inteligente de Lisboa (PGIL) com vista à supervisão dos vários serviços do município da capital e para a tomada de decisão em tempo real, evidenciando um exemplo de passagem de uma estratégia de primeira geração para uma de segunda geração.

De acordo com os rankings sobre smart cities disponíveis, no Brasil, os municípios em melhor posição estão localizados nas capitais e regiões mais desenvolvidas do país. Em Portugal, o principal índice, nas suas duas versões, apresentou, no primeiro ano, uma concentração de municípios das duas áreas metropolitanas e em cidades universitárias, e, em 2016, prevalência de municípios do norte do país, especificamente em cidades médias.

Quanto aos setores em que se centram os projetos, a iluminação pública e a mobilidade parecem ser áreas de destaque. Tanto Portugal como Brasil têm priorizado investimentos em iluminação pública inteligente, no primeiro caso motivada pela existência de fundos europeus para o efeito e, no segundo, devido a um imperativo legal que obriga as municipalidades a modernizarem o serviço. Ou seja, em ambos o motivador inicial não tem sido a melhoria da qualidade de vida dos residentes, embora a população possa ser beneficiada de forma colateral, seja com a melhoria do serviço em si ou com a poupança de recurso com um serviço mais eficiente.

Na mobilidade inteligente, Portugal surge com mais iniciativas, não só de mobilidade elétrica, mas também nas mobilidades suaves. Uma diversidade de plataformas surgiu nos últimos anos competindo entre si pelo mercado urbano da mobilidade suave através de bicicletas e trotinetes. Trata-se, em todo o caso, de processos em estádios iniciais, para já ainda com maior atração para o turista do que para o habitante e requerendo, portanto, consolidação.

Quanto aos constrangimentos existentes, o financiamento dos projetos parece ser crucial nos dois países. No Brasil há exemplos de investimentos financiados por empresas privadas com o objetivo de vender os seus serviços e produtos a outros municípios. Esta situação, contudo, não é a ideal, pois limita o poder de atuação do gestor público e tem como principal motivador a realização de lucros para o setor privado, sendo que os efeitos positivos passam a ser efeitos secundários do processo. Com a atual crise fiscal, o uso de orçamentos próprios para a contratação de projetos passa a ser ainda mais difícil no Brasil. Mesmo com o aumento de PPP, o país ainda tem fragilidades técnicas importantes que impedem um avanço maior no uso desse tipo de instrumento. A possibilidade de recorrer a fundos europeus no financiamento de projetos pode ser mais favorável para Portugal. Mesmo que o tema não tenha ainda sido colocado de forma central no planejamento europeu, a conscientização dos governos locais sobre a sua importância parece indicar que o assunto poderá ser mais valorizado nas rondas de planejamento futuras, sendo um maior racional estratégico regional uma das questões a ter em atenção.

\section{Referências}

Agência Nacional de Energia Elétrica - ANEEL. (2010, 9 de setembro). Resolução Normativa nº 414, de 9 de setembro de 2010. Estabelece as Condições Gerais de Fornecimento de Energia Elétrica de forma atualizada e consolidada. Recuperado em 9 de maio de 2019, de

http://www.aneel.gov.br/documents/656877/14486448/bren2010414.pdf/3bd33297-26f9-4ddf-94c3-

f01d76d6f14a?version=1.0 
Agência Nacional de Energia Elétrica - ANEEL. (2012, 3 de abril). Resolução Normativa no 479, de 3 de abril de 2012. Altera a Resolução Normativa no 414, de 9 de setembro de 2010, que estabelece as Condições Gerais de Fornecimento de Energia Elétrica de forma atualizada e consolidada. Recuperado em 9 de maio de 2019, de http://www2.aneel.gov.br/aplicacoes/audiencia/arquivo/2011/049/resultado/ren2012479.pdf

Agência Nacional de Energia Elétrica - ANEEL. (2017). Brasil possui mais de 10 mil conexões de micro e minigeração distribuída. Recuperado em 23 de abril de 2019, de http://www.aneel.gov.br/sala-de-imprensa-exibicao//asset_publisher/XGPXSqdMFHrE/content/brasil-possui-mais-de-10-mil-conexoes-de-micro-e-minigeracaodistribuida/656877?inheritRedirect=false

Agência Nacional de Energia Elétrica - ANEEL. (2018). Geração distribuída: contribuições para consulta pública cp 10/2018: aprimoramento das regras aplicáveis à micro e minigeração distribuída.Resolução Normativa 482/2012 ANEEL. Brasília.

Agência Nacional de Telecomunicações - ANATEL. (2018, 11 de setembro). Anatel abre Tomada de Subsídio para simplificar regulamentação para Internet das Coisas. Recuperado em 9 de maio de 2019, de http://www.anatel.gov.br/institucional/ultimas-noticiass/2067-anatel-abre-tomada-de-subsidio-parasimplificar-regulamentacao-para-internet-das-coisas

Agência Nacional de Vigilância Sanitária - ANVISA. (2019). Análise de impacto regulatório. Recuperado em 23 de abril de 2019, de http://portal.anvisa.gov.br/analise-de-impacto-regulatorio

Albino, V., Berardi, U., \& Dangelico, R. M. (2015). Smart Cities: definitions, dimensions, performance and initiatives. Journal of Urban Technology, 22(1), 3-21. http://dx.doi.org/10.1080/10630732.2014.942092.

Banco Nacional de Desenvolvimento Econômico e Social - BNDES. (2018). Internet das coisas: um plano de ação para o Brasil (Cartilha de Cidades). Rio de Janeiro. Recuperado em 23 de janeiro de 2019, de http://www.abinee.org.br/informac/arquivos/bndes2.pdf

Bencardino, M., \& Greco, I. (2014). Smart communities: social innovation at the service of the smart cities. Journal of Land Use, Mobility and Environment, 39-51. http://dx.doi.org/10.6092/1970-9870/2533.

Black \& Veatch. (2016). 2016 strategic directions: U.S. smart city/smart utility report. Recuperado em 13 fevereiro 2019, de https://www.bv.com/sites/default/files/gated-content/strategic-directions-report/18-SDR-SmartCities-Utilities.pdf

Cohen, B. (2015) The 3 generations of Smart Cities: inside the development of the technology driven city. Recuperado em 20 fevereiro de 2019, de https://www.fastcompany.com/3047795/the-3-generations-of-smart-cities

Connected Smart Cities. (2015). Recuperado em 21 fevereiro de 2019, de

https://issuu.com/connectedsmartcities/docs/cat_logo_connected_smart_cities_20

Connected Smart Cities. (2016). Recuperado em 24 fevereiro de 2019, de http://www.connectedsmartcities.com.br/resultados-do-ranking-connected-smart-cities/\#

Cunha, M., Przeybilovicz, E., Macaya, J., \& Burgos, F. (2016). Smart City: transformação digital de cidades. São Paulo: Programa Gestão Pública e Cidadania.

Dias, R. C., \& Seixas, P. C. (2018). Modelos regionais de governança da sustentabilidade: uma análise às primeiras estratégias integradas de desenvolvimento regional em Portugal. Revista Portuguesa de Estudos Regionais, (48), 5-16.

Duarte, F., \& Firmino, R. (2011). Da coisa ao objeto, do artefato à tecnologia ubíqua. Com Ciência: Revista Eletrônica de Jornalismo Científico. Recuperado em 1 de março de 2019, de

http://www.comciencia.br/comciencia/handler.php?section=8\&edicao=70\&id=867

Dutton, W. H., Blumber, J. G., \& Kraemer, K. L. (1987). Wired cities: shaping future communication. New York: Macmillan.

Ferreira, F., \& Seixas, P. C. (2017). Portugal 2020 e o novo glossário de desenvolvimento territorial: territorialização ou neoinstitucionlização? urbe. Revista Brasileira de Gestão Urbana, 9(3), 487-499. http://dx.doi.org/10.1590/2175-3369.009.003.ao08. 
Frey, K., Kauchakje, S., Penna, M. C., Duarte, F., \& Procupiuck, M. (2007). Redes sociotécnicas e políticas públicas. In T. T. C. Egler (Ed.), Ciberpólis: redes no governo da cidade (pp. 85-97). Rio de Janeiro: 7Letras.

Goodspeed, R. (2015). Smart cities: moving beyond urban cybernetics to tackle wicked problems. Cambridge Journal of Regions, Economy and Society, 8(1), 79-92. http://dx.doi.org/10.1093/cjres/rsu013.

Graham, S., \& Marvin, S. (1999). Planning cyber cities: integrating telecommunications into urban planning. The Town Planning Review, 70(1), 89-114. http://dx.doi.org/10.3828/tpr.70.1.w34454x3475g2858.

INTELI. (2012). Índice de Cidades Inteligentes Portugal. Lisboa.

INTELI. (2016). Índice de Cidades Inteligentes Portugal. Lisboa.

INTELI. (2019a). Primeiro Living Lab de Mobilidade na Europa. Recuperado em 20 fevereiro de 2019, de http://www.inteli.pt/pt/go/rener1

INTELI. (2019b). A Smart Project for Smart Cities. Recuperado em 3 de fevereiro de 2019, de http://www.inteli.pt/pt/go/a-smart-project-for-smart-cities

International Telecommunication Union - ITU.(2014). ITU-T focus group on smart sustainable cities: smart sustainable cities: an analysis of definitions. Focus Group Technical Report. Recuperado em 12 de fevereiro de 2019, de https://www.itu.int/en/ITU-T/.../ssc/.../TR-Definitions.docx

Ishida, T., \& Isbister, K. (2000). Digital cities: technologies, experiences, and future perspetives. Berlin: Springer. http://dx.doi.org/10.1007/3-540-46422-0.

ITF365. (2017). Algar Telecom levará conceito de cidade inteligente para bairro de Uberlândia. Recuperado em 7 de fevereiro de 2019, de https://www.itforum365.com.br/tecnologia/algar-telecom-levara-conceito-de-cidadeinteligente-para-bairro-de-uberlandia/

Komninos, N. (2002). Intelligent cities: innovation, knowledge systems and digital spaces. Abington: Routledge.

Leem, C. S., \& Kim, B. G. (2013). Taxonomy of ubiquitous computing service for city development. Personal and Ubiquitous Computing, 17(7), 1475-1483. http://dx.doi.org/10.1007/s00779-012-0583-5.

Mobi.E. (2019). Onde utilizar a rede Mobi.E? Recuperado em 20 de fevereiro de 2019, de https://www.mobie.pt/where-electric-mobility

Mora, L., Bolici, R., \& Deakin, M. (2017). The first two decades of smart-city research: a bibliometric analysis. Journal of Urban Technology, 24(1), 3-27. http://dx.doi.org/10.1080/10630732.2017.1285123.

Przeybilovicz, E., Cunha, M. A., \& Quandt, C. O. (2014). 0 perfil dos municípios brasileiros em relação ao uso e à infraestrutura de TIC: uma análise dos clusters. In Anais do XXXVIII Encontro da ANPAD. Rio de Janeiro: ANPAD. Recuperado em 23 de abril de 2019, de http://www.anpad.org.br/admin/pdf/2014_EnANPAD_ADI2033.pdf

Público. (2016). As cidades inteligentes ainda estão na "fase-piloto". Recuperado em 10 de fevereiro de 2019, de https://www.publico.pt/2016/03/06/local/noticia/em-dez-anos-as-cidades-inteligentes-nao-sairam-da-fasepiloto-em-portugal-1725220

Rede Brasileira de Cidades Inteligentes e Humanas. (2019). Projeto de Cidades Inteligentes do Sebrae Nacional seleciona Campina Grande. Recuperado em 9 de maio de 2019, de http://redebrasileira.org/materias/2934/projetode-cidades-inteligentes-do-sebrae-nacional-seleciona-campina-grande

Robinson, R. (2016). Why Smart Cities still aren't working for us after 20 years: and how we can fix them. Recuperado em 8 de janeiro de 2019, de https://theurbantechnologist.com/2016/02/01/why-smart-cities-stillarent-working-for-us-after-20-years-and-how-we-can-fix-them/

SapoTek. (2018). Há um cluster de smart cities em desenvolvimento em Portugal mas precisa de financiamento. Recuperado em 11 de fevereiro de 2019, de https://tek.sapo.pt/noticias/negocios/artigos/ha-um-cluster-desmart-cities-em-desenvolvimento-em-portugal-mas-precisa-de-financiamento

Seixas, P. C. (2019). Introdução. In: P. C. Seixas (Ed.), Ativar cidades: modelos de políticas de cidades (pp. 7-11. Lisboa: Caleidoscópio. 
Seixas, P. C., Dias, R. C., Pereira, P. (2017). Uma cidade boa para viver: planeamento cultural e ciência cidadã no desenvolvimento urbano sustentável. A Obra Nasce, 12, 9-25.

Selada, C. (2019). A Cidade Inteligente: um ecossistema urbano de inovação. In: P. C. Seixas (Ed.), Ativar cidades: modelos de políticas de cidades (pp. 102-113). Lisboa: Caleidoscópio.

Selada, C., \& Silva, C. (2013). As Cidades Inteligentes na Agenda Europeia: oportunidades para Portugal. In Anais do II Conferência de PRU, VIII ENPLAN e XVIII Workshop APDR: Europa 2020: retórica, discursos, política e prática. Lisboa: INTELI. Recuperado em 12 de janeiro de 2019, de http://www.inteli.pt/uploads/documentos/documento_1373454640_1255.pdf

Smart City Hub. (2018). PlanIT Valley: the smartest city never been built. Recuperado em 14 janeiro de 2019, de http://smartcityhub.com/governance-economy/planit-valley-the-smartest-city-never-been-built/

Smart City Laguna. (2019). O empreendimento. Recuperado em 20 fevereiro de 2019, de http://smartcitylaguna.com.br/empreendimento/

Telefônica. (2015). Águas de São Pedro, a model to follow for all Brazil. Recuperado em 12 de fevereiro de 2019, de https://iot.telefonica.com/blog/aguas-de-sao-pedro-a-model-to-follow-for-all-brazil

Telesíntese. (2011). Belo Horizonte, Curitiba e Porto Alegre. As três melhores colocadas no índice Brasil de Cidades Digitais. Recuperado em 12 de fevereiro de 2019, de http://www.telesintese.com.br/belo-horizonte-curitiba-eporto-alegre-as-tres-melhores-colocadas-no-indice-brasil-de-cidades-digitais/

Telesíntese. (2012). Curitiba fica em 1ํo lugar no índice de Cidades Digitais 2012. Recuperado em 10 de fevereiro de 2019, de http://www.telesintese.com.br/curitiba-fica-em-1-no-indice-de-cidades-digitais-2012/

Torres Vedras. Câmara Municipal. (2013). Bicicletas públicas de Torres Vedras estão disponíveis. Recuperado em 10 de fevereiro de 2019, de http://www.cm-tvedras.pt/artigos/detalhes/bicicletas-publicas-de-torres-vedras-estao-disponiveis/

Editores: Janaina Camile Pasqual Lofhagen, Pontifícia Universidade Católica do Paraná - PUCPR; Christopher Hawkins, University of Central Florida - UCF

Recebido: Mar. 13, 2019

Aprovado: Abr. 24, 2019 